\title{
Molecular analysis of contiguous exons of the phenylalanine hydroxylase gene: identification of a new PKU mutation
}

\author{
I Dianzani, C Camaschella, G Saglio, G B Ferrero, S Ramus, A Ponzone, \\ R G H Cotton
}

\begin{abstract}
A modified application of the chemical cleavage of mismatch (CCM) method has been used to screen three contiguous exons (exons 9, 10, and 11) of the phenylalanine hydroxylase gene in 17 Italian PKU patients. A new nonsense heterozygous $C \rightarrow G$ transversion within exon 11 (S359X) was identified in a single patient. Only one of the four mutations previously reported in this DNA region in Caucasians was found. This lesion, IVS X-546, was detected in five of the 34 PKU alleles examined. Our results underline the versatility of the CCM method for scanning a gene for multiple mutations. ( $\mathcal{F}$ Med Genet 1993;30:228-31)
\end{abstract}

Phenylketonuria (PKU) is an inborn error of metabolism owing to deficiency of the liver enzyme phenylalanine hydroxylase $(\mathrm{PAH}) .{ }^{1} \mathrm{In}$ the case of large genes with tissue specific expression like PAH, the unavailability of the correct tissue is a drawback to rapid identification of the causal mutations. ${ }^{2}$ Usually, single exons have to be screened for mutations. A number of methods for mutation detection have recently been proposed as an alternative to extensive sequencing. The most efficient methods are those able to screen both for known and new mutations, such as denaturing gradient gel electrophoresis (DGGE), ${ }^{3}$ chemical cleavage of mismatch (CCM) ${ }^{4}$ and single strand conformation polymorphism (SSCP). ${ }^{5}$ All these methods eventually require sequence analysis for identification of the exact DNA change.

The possibility of studying multiple exons in the same assay speeds up the screening, as shown by the use of multiplex PCR analysis. ${ }^{6}$

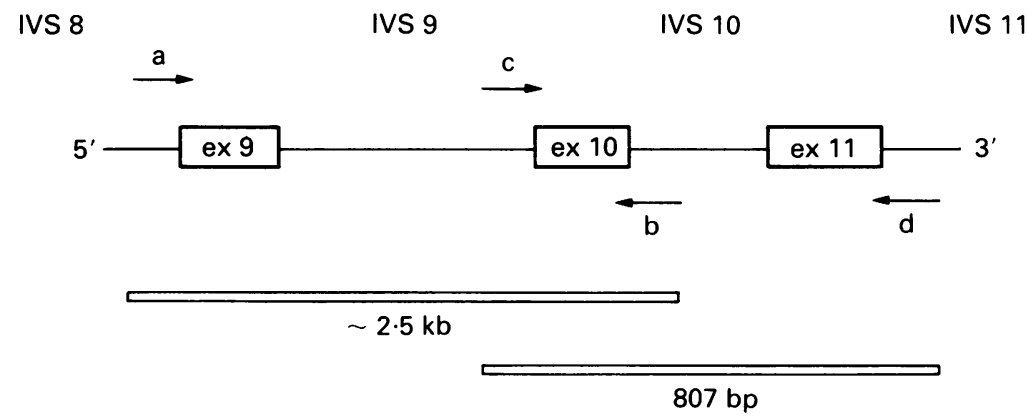

Figure 1 Schematic representation of the PCR products obtained using primers a and $b, c$ and $d$.
We have recently used CCM to analyse simultaneously two contiguous exons (exons 7 and 8) of phenylalanine hydroxylase (PAH), ${ }^{7}$ taking advantage of the ability of CCM to screen large DNA fragments. We report here the screening of three other exons $(9,10$, and 11) of the PAH gene from Italian PKU patients. A new mutation was identified in a single patient and a recently reported mutation, specific to Mediterranean PKU, ${ }^{89}$ was observed in several patients.

\section{Materials and methods \\ PATIENTS}

Seventeen unrelated Italian PKU patients were investigated. The patients originated from different regions in Italy and were thus a representative section of the whole Italian population. RFLP haplotype determination was available for nine patients. ${ }^{10}$ Genomic DNA from patients and normal controls was extracted from peripheral blood leucocytes by standard techniques.

\section{PCR AMPLIFICATION OF PAH EXONS}

Exons 9 and 10 of the PAH gene were amplified in a single polymerase chain reaction (PCR) using appropriate primers corresponding to the intron-exon boundaries, namely of intron 8-exon 9 (primer a, 5'-TTTTCCCCCAATTACAGGAA- $3^{\prime}$ ) and of exon 10-intron 10 (primer b, 5'-AAGGTCATACCTGTAATTCA-3'), ${ }^{11}$ as shown in fig 1 . The length of the PCR product was approximately $2.5 \mathrm{~kb}$ (unavailability of the complete sequence of intron 9 allowed only an approximate estimate; for the same reason PCR primers were designed at intron-exon boundaries).

Exons 10 and 11 were amplified using a $5^{\prime}$ primer (primer c, $5^{\prime}$-ATTGACTTTCCATCCAGATT-3') complementary to the intron 9exon 10 boundary and a $3^{\prime}$ primer (primer d, 5'-ACCACCTCACCTTACTTTCT-3') complementary to the exon 11 -intron 11 boundary. ${ }^{11}$ Since the length of intron 10 is $556 \mathrm{bp},{ }^{89}$ the PCR product was $807 \mathrm{bp}$ (fig 1).

PCR was performed using the Cetus Perkin Elmer PCR protocol using the following conditions: denaturation $\left(105\right.$ seconds at $95^{\circ} \mathrm{C}$ ), annealing $\left(120\right.$ seconds at $\left.65^{\circ} \mathrm{C}\right)$, and extension $\left(180\right.$ seconds at $\left.72^{\circ} \mathrm{C}\right)$ for 35 cycles. In both reactions the final $\mathrm{MgCl}_{2}$ concentration was $2.5 \mathrm{mmol} / 1$.

The study of overlapping genomic fragments allowed us to screen all intron-exon 
boundaries with the exception of intron 8-exon 9 and exon 11-intron 11 boundaries.

\section{CCM ANALYSIS}

Two different sets of experiments were performed: the first (experiment A) using PCR products encompassing exons $9-10$ and the second (experiment B) using PCR products encompassing exons 10-11.

For each experiment, a uniformly labelled probe was obtained using DNA from a normal control. Probe labelling, purification of the labelled PCR product, heteroduplex production, and CCM reactions were performed as previously reported. ${ }^{7}$

The screening of overlapping DNA fragments increases the information obtained by using a uniformly labelled probe. Comparison of the two experiments allows localisation of mismatches within one of the two ends of the molecule in each reaction. Consequently, sequencing is reduced. The small regions corresponding to primers $\mathrm{b}$ and $\mathrm{c}$ at the boundaries of exon 10 are not included in the overlap, as mutations at primer sites are not detected.

\section{ASO AND SEQUENCE ANALYSIS}

Amplified PCR products were directly sequenced using the Sequenase DNA

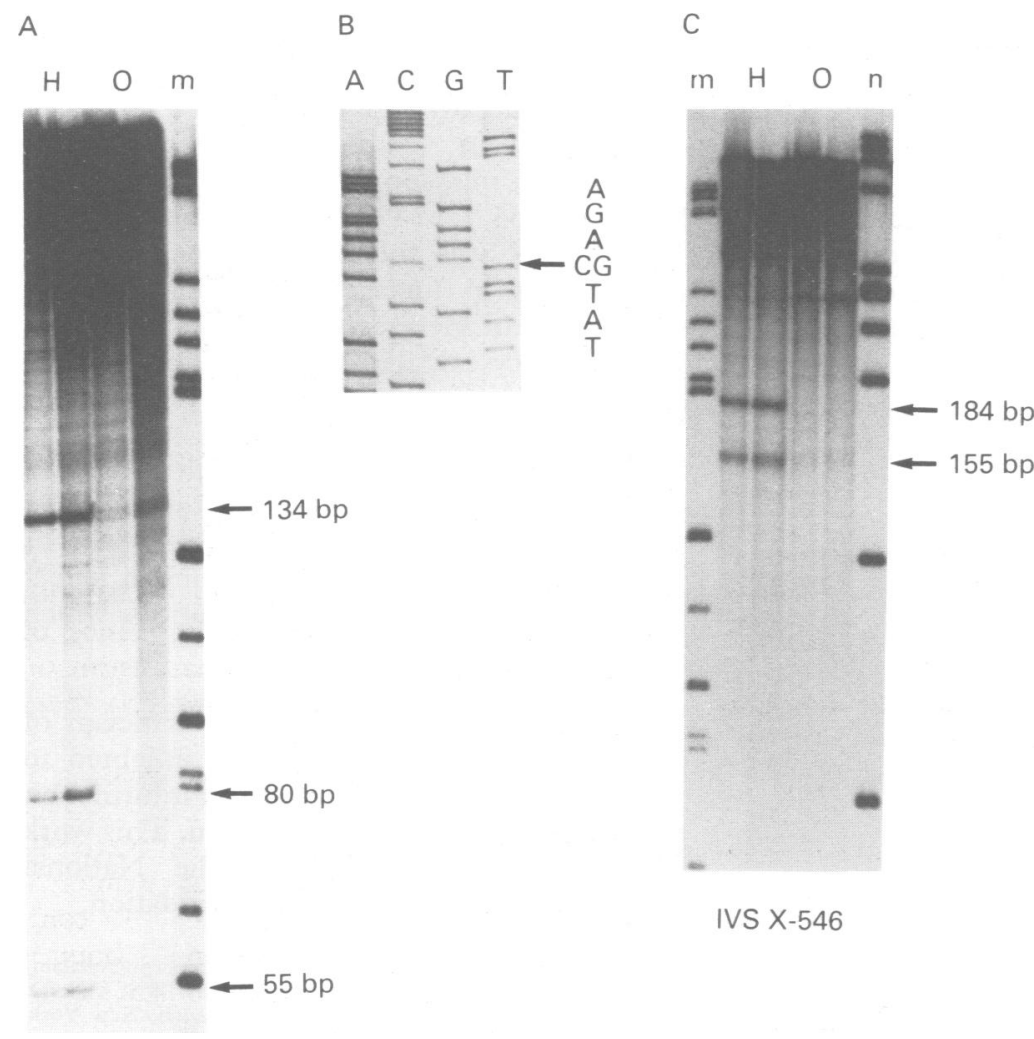

Figure 2 ( $A$ ) CCM analysis of PCR fragments encompassing $P A H$ exons 10 and 11 from an Italian PKU patient, showing mismatches at 134, 80, and 55 bp. $H$, hydroxylamine; $O$, osmium tetroxide; $m$, marker ladder. Mismatches at 55 and $80 \mathrm{bp}$ are generated by a $G \rightarrow C$ polymorphism at codon 385 within the probe (see text). (B Direct sequence analysis of a portion of the $807 \mathrm{bp} P C R$ product from the same patient in the area corresponding to the mismatch at $134 \mathrm{bp}$. The mutant shows $a C \rightarrow G$ transition at position 359. (C) CCM analysis of PCR fragments encompassing $P A H$ exons 10 and 11 from an Italian PKU patient homozygous for haplotype $6 . H$, hydroxylamine; $O$, osmium tetroxide; $m$ and $n$, marker ladders. The mismatch at 155 bp represents the CCM pattern of mutation IVS X-546. Mismatch at 184 represents a polymorphism linked to IVS X-546. sequencing kit (United States Biochemical Corporation) and a protocol reported previously. ${ }^{12}$ ASO analysis was performed using oligonucleotides complementary to the mutated sequences of the two identified mutations. Amplified DNA from 130 PKU Italian alleles were used to identify the frequency of both mutations within the Italian population.

\section{Results}

CCM AND SEQUENCE ANALYSIS

Experiment A did not show mismatches within exons 9 and 10 of PAH from the PKU subjects, whereas experiment $B$ showed two specific patterns of mismatches. The first was observed in a single patient and included three bands, mapping approximately at 140,80 , and $55 \mathrm{bp}$ (fig $2 \mathrm{~A}$ ). Sequence analysis identified a $\mathrm{C} \rightarrow \mathrm{G}$ transversion mapping at position 134 of the PCR fragment (fig 2B). This mutation changes the TCA codon for serine 359 into a stop codon within exon 11 (S359X). We did not find any change corresponding to mismatches at 80 and $55 \mathrm{bp}$ in the DNA from this patient.

However, sequence analysis of the control DNA used as a probe identified a recently reported $\mathrm{G} \rightarrow \mathrm{C}$ polymorphism at base $1377^{2}$ (along with the normal sequence), corresponding to coilon 385 and not to codon 430 as was previously reported (S Forrest, personal communication)

The presence of this polymorphism in the control DNA and of the mutation at codon 359 in the PKU patient, both changes in heterozygosity with the normal sequence, ${ }^{13}$ account for the three mismatches identified in the CCM reaction. In fact, three of all the possible heteroduplexes generated in this reaction are expected to react with hydroxylamine. One of them detects only the S359X mutation as a band at $134 \mathrm{bp}$, another only the codon 385 polymorphism as a band at $55 \mathrm{bp}$, whereas the third detects both changes and generates bands at 55 and $80 \mathrm{bp}$.

The second pattern of mismatch was represented by fragments at 184 and at $155 \mathrm{bp}$ (fig 2C) and was seen in four patients. Since no mismatch was identified in the same patients when exons 9-10 were investigated, we assumed that the corresponding DNA changes were located close to exon 11 (exon 11 is $134 \mathrm{bp}$ long). Since one out of the four patients showing the mismatch at $155 \mathrm{bp}$ was homozygous for haplotype 6 , we assumed that the 155 bp mismatch represented the CCM pattern of a common Mediterranean PKU mutation mapping 11 nucleotides before exon 11 and in linkage disequilibrium with haplotype 6 (IVS X-546, G $\rightarrow \mathrm{A}) .{ }^{8914}$ This hypothesis was confirmed by hybridisation of the DNA from all the 17 PKU patients with an ASO complementary to the IVS X-546 mutation (see below).

In all the four subjects the IVS X-546 mutation seems to be linked with a $184 \mathrm{bp}$ mismatch. Since this mismatch was found in two PKU patients in which both PKU mutations had been completely characterised, the 
corresponding DNA change may be considered to be a harmless linked polymorphism.

\section{ASO ANALYSIS}

Hybridisation with an ASO complementary to the S359X mutation (5'-TACTGCTTATGAGAGAAGCC-3') on PCR products, obtained by using primers $c$ and $d$ and DNA from the 17 PKU patients, confirmed the data obtained by CCM analysis (fig 3B). ASO analysis of PCR products from the parents of the single patient carrier of S359X showed that the mutation was inherited from the mother (fig 3B). Twenty normal subjects similarly screened were proven not to carry the molecular lesion (data not shown).

Hybridisation with an ASO complementary to the IVS X-546 mutation ${ }^{9}$ on the same PCR products used above confirmed the data obtained by CCM analysis: only the four patients who showed the mismatch at $155 \mathrm{bp}$ hybridised with the mutated ASO (fig 3A).

Analysis of 110 Italian PKU alleles by using ASOs complementary to both mutations allowed us to evaluate the frequency of these DNA changes within the Italian population: $0.8 \%$ for S359X and $10 \%$ for IVS X-546.

\section{Discussion}

Recent studies have suggested a different molecular basis for PKU in northern and southern Europe. The existence of a peculiar pattern of mutations in Italian PKU was first assumed on the basis of a different RFLP haplotype distribution and by the rarity of the two mutations that are most frequent in northern European PKU. ${ }^{10}$ Molecular heterogeneity was inferred both by haplotype distribution and by the association of different clinical phenotypes with the same haplotype.

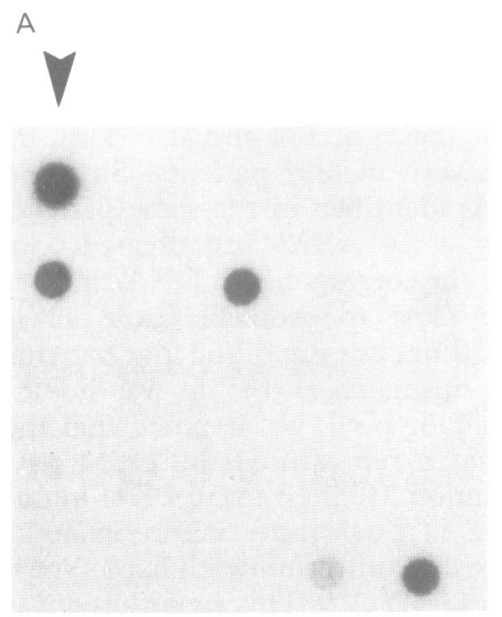

IVS $\times-546$

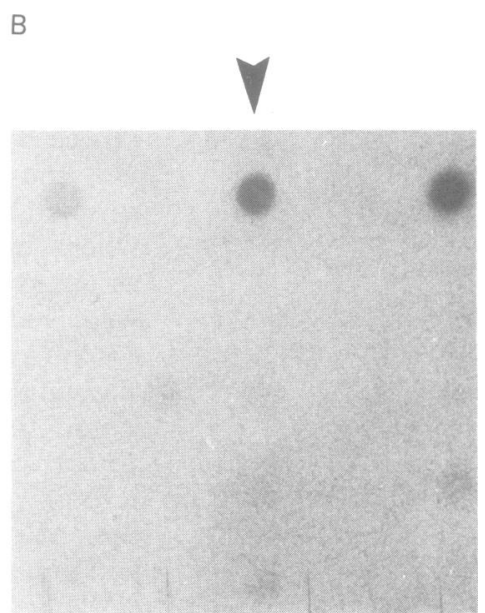

S359X
Figure 3 Dot blot analysis of the IVS X-546 (A) and S359X (B) mutations in Italian PKU subjects. A sample of the 130 screened chromosomes is represented. The same filter was hybridised consecutively with oligonucleotides complementary to each of the two mutations. Exons 10-11 of PAH were amplified by using appropriate primers (see text). The positive sample on the upper left of $(A)$ (arrow) corresponds to the single patient homozygous for IVS X-546. The other positive samples in $(A)$ correspond to the three remaining patients previously identified by CCM. The two positive samples in $(B)$ represent the single patient carrier of the $S 359 X$ mutation (arrow) and his heterozygous mother.
Recently, this hypothesis has been confirmed by the identification of several mutations peculiar to Mediterranean populations and by the rarity of the many mutations identified in northern Europeans. ${ }^{15}$ However, the overall frequency of the seven mutations so far identified among Mediterraneans (IVS X546, ${ }^{89}$ R261Q, ${ }^{16}{ }^{17}$ R252W, ${ }^{1618}$ P281L, ${ }^{18}$ IVS VII- $1,{ }^{7}$ R408W, ${ }^{19}$ IVS XII- ${ }^{20}$ ) still only accounts for approximately $40 \%$ of the Italian PKU alleles.

We were thus prompted to search for other causal mutations in Italian PKU. We focused our attention on the $3^{\prime}$ region of the $\mathrm{PAH}$ gene because it encodes a portion of the protein essential for catalytic activity. ${ }^{21} 22$ In a previous study we screened a panel of Italian PKU patients for all the DNA changes which mapped within exons $7-8,{ }^{7}$ the region which encodes the putative cofactor binding site for PAH. ${ }^{23}$ In this study we screened exons 9,10 , and 11.

A new mutation (S359X) was identified within exon 11 in a single PKU patient. Since it is a termination mutation, the mutated gene encodes for a truncated protein, lacking the region corresponding to exons 12 and 13 and most of exon 11. This region is essential for enzyme function, as shown by the absence of enzymatic activity of transformed cells containing another mutated PAH allele (IVS XIII), which express a truncated protein lacking the C-terminus portion corresponding to exons 12 and $13 . .^{24}$ Thus, the S359X mutation is likely to be causal. Four causal mutations have been already reported to affect exons 9 , 10, and 11 in Caucasians and were thus screened by our approach (L311P, ${ }^{25}$ IVS X$546,{ }^{89}$ del364, ${ }^{26} \mathrm{~S} 349 \mathrm{R}^{2}$ ). Only one of these, IVS X-546, was found in our series. This mutation is in linkage disequilibrium with haplotype 6 in Mediterranean PKU patients. ${ }^{89}$ Its frequency calculated for 130 Italian PKU alleles $(10 \%)$ corresponds to that reported for haplotype $6 .^{10}$

These findings further support the existence of molecular heterogeneity within Italian PKU. Such molecular heterogeneity might have originated through a founder effect within the multiple ethnic populations which represented Italy in the past.

Dr S M Forrest is thanked for criticism of the manuscript. We are greatly indebted to Dr S L C Woo for prepublication information regarding the IVS X-546 mutation. This work was partially supported by the National Australian Medical Research Foundation.

1 Scriver CR, Kaufman S, Woo SLC. The hyperphenylalaninemias. In: Scriver CR, Beaudet AL, Sly WS, Valle D, nemias. In: Scriver CR, Beaudet AL, Sly WS, Valle D, eds. The metabolic basis of in

2 Forrest SM, Dahl HH, Howells DW, Dianzani I, Cotton RGH. Mutation detection in phenylketonuria using the chemical cleavage of mismatch method: importance of using probes from both normal and patient samples. $A m \mathcal{J}$ Hum Genet 1991;49:175-83.

3 Myers RM, Maniatis T, Lerman LS. Detection and localzation of single base changes by denaturing gradient gel electrophoresis. Methods Enzymol 1987;155:501-27.

4 Cotton RGH, Rodrigues NR, Campbell RD. Reactivity of cytosine and thymine in single-base-pair mismatches with hydroxylamine and osmium tetroxide and its application to the study of mutations. Proc Natl Acad Sci USA 1988;85:4397-401. 
5 Orita M, Suzuki Y, Sekiya T, Hayashi K. Rapid and sensitive detection of point mutations and DNA polymor-
phisms using the polymerase chain reaction. Genomics phisms using

6 Chamberlain JS, Gibbs RA, Ranier JE, Nguyen PN, Caskey CT. Deletions screening of the Duchenne muscula dystrophy locus via multiplex DNA amplification. Nucleic Acids Res 1988;16:11141-56.

7 Dianzani I, Forrest SM, Camaschella C, Saglio G, Ponzone A, Cotton RGH. Screening for PKU mutations in the phenylalanine hydroxylase gene from Italian patients with phenylketonuria using the chemical cleavage method: a new splice mutation. Am f Hum Genet 1991;48:631-5.

8 Kalaydjieva L, Dworniczak B, Aulehla-Scholz C, et al. Phenylketonuria mutation in southern Europeans. Lancet 1991;337:865.

9 Dasovich M, Konecki D, Lichter-Konecki U, et al. Molecular characterization of a PKU allele prevalent in Southcular characterization of a PKU allele prevalent in Southern Europe

10 Dianzani I, Devoto M, Camaschella C, et al. Haplotype distribution and molecular defects at the phenylalanine hydroxylase locus in Italy. Hum Genet 1990;86:69-72.

11 DiLella AG, Kwok SCM, Ledley FD, Marvit J, Woo SLC. Molecular structure and polymorphic map of the human phenylalanine hydroxylase gene. Biochemistry 1986;25:743-8.

12 Dianzani I, Camaschella C, Saglio G, Forrest SM, Ramus $\mathrm{S}$, Cotton RGH. Simultaneous screening for $\beta$ thalassemia by chemical cleavage of mismatch Genomics 1991;11:48-53.

13 Dianzani I, Forrest SM, Camaschella C, Gottardi E, Cotton RGH. Heterozygotes and homozygotes discrimination by chemical cleavage of mismatch. Am $\mathcal{f}$ Hum Genet 1991;48:423-4.

14 Dworniczak B, Aulehla-Scholz C, Kalaydiieva L, Bartholome K, Grudda K, Horst J. Aberrant splicing of phenylalanine hydroxylase mRNA: the major cause for phenylketonuria in parts of Southern Europe. Genomics 1991;11:242-6.

15 Dianzani I, Camaschella C, Ferrero GB, Cotton RGH, Ponzone A. Molecular basis of PKU in Italy. Pteridines 1992;3:11-12.
16 Abadie V, Lyonnet S, Maurin N, et al. CpG dinucleotides are mutation hot spots in phenylketonuria. Genomics 1989;5:936-9.

17 Okano Y, Wang T, Eisensmith RC, Steinmann B, Gitzelmann R, Woo SLC. Missense mutations associated with RFLP haplotypes 1 and 4 of the phenylalanine hydroxylase gene. Am $\mathcal{F}$ Hum Genet 1990;46:18-25.

18 Okano Y, Wang T, Eisensmith RC, et al. Phenylketonuria missense mutations in the Mediterranean. Genomics 1991;9:93-103.

19 DiLella AG, Marvit J, Brayton K, Woo SLC. An aminoacid substitution involved in phenylketonuria is in linkage disequilibrium with DNA haplotype 2. Nature 1987; 327:333-6.

20 DiLella AG, Marvit J, Lidski AS, Guttler F, Woo SLC Tight linkage between a splicing mutation and a specific DNA haplotype in phenylketonuria. Nature 1986; 322:799-803.

21 Iwaki M, Phillips RS, Kaufman S. Proteolytic modification of the amino-terminal and carboxyl-terminal regions of
rat hepatic phenylalanine hydroxylase. $f$ Biol Chem rat hepatic phen

22 Grenett HE, Ledley FD, Reed LL, Woo SLC. Full length cDNA for rabbit tryptophan hydroxylase: functional domains and evolution of aromatic aminoacid hydroxylases. Proc Natl Acad Sci USA 1987;84:5530-4.

23 Jennings IG, Kemp BE, Cotton RGH. Localisation of cofactor binding sites with monoclonal anti-idiotype antibodies: phenylalanine hydroxylase. Proc Natl Acad Sci USA 1991;88:5734-8.

24 Marvit J, DiLella AG, Brayton K, Ledley FD, Robson KJ Woo SLC. GT to AT transition at a splice donor site causes skipping of the preceding exon in phenylketonuria. causes skipping of the preceding exo

25 Lichter-Konecki U, Konecki DS, DiLella AG, et al. Phenylalanine hydroxylase deficiency caused by a single base substitution in an exon of the human phenylalanine substitution in an exon of the human phen
hydroxylase gene. Biochemistry 1988;27:2881-5.

26 Svensson E, Andersson B, Hagenfeld L. Two mutations within the coding sequence of the phenylalanine hydroxy-
witans lase gene. Hum Genet 1990;85:300-4. 